\title{
Approximation Algorithm for Non-Boolean MAX $k$-CSP
}

\author{
Konstantin Makarychev ${ }^{1}$ and Yury Makarychev ${ }^{2 \star}$ \\ ${ }^{1}$ Microsoft Research \\ 2 Toyota Technological Institute at Chicago
}

\begin{abstract}
In this paper, we present a randomized polynomial-time approximation algorithm for MAX $k$-CSP ${ }_{d}$. In MAX $k$-CSP ${ }_{d}$, we are given a set of predicates of arity $k$ over an alphabet of size $d$. Our goal is to find an assignment that maximizes the number of satisfied constraints.

Our algorithm has approximation factor $\Omega\left(k d / d^{k}\right)($ when $k \geq \Omega(\log d))$. This bound is asymptotically optimal assuming the Unique Games Conjecture. The best previously known algorithm has approximation factor $\Omega\left(k \log d / d^{k}\right)$.

We also give an approximation algorithm for the boolean MAX $k$-CSP 2 problem with a slightly improved approximation guarantee.
\end{abstract}

\section{Introduction}

We design an approximation algorithm for the MAX $k-\mathrm{CSP}_{d}$, the maximum constraint satisfaction problem with $k$-ary predicates and domain size $d$. In this problem, we are given a set of variables $\left\{x_{u}\right\}_{u \in X}$ and a set of predicates $\mathcal{P}$. Each variable $x_{u}$ takes values in $[d]=\{1, \ldots, d\}$. Each predicate $P \in \mathcal{P}$ depends on at most $k$ variables. Our goal is to assign values to variables so as to maximize the number of satisfied constraints.

There has been a lot of interest in finding the approximability of MAX $k$-CSP $d$ in the complexity community motivated by the connection of MAX $k$-CSP $d$ to $k$-bit PCPs. Let us briefly overview known results. Samorodnitsky and Trevisan [9] showed that the boolean MAX $k$-CSP $\mathrm{C}_{2}$ problem cannot be approximated within a factor of $\Omega\left(2^{2 \sqrt{k}} / 2^{k}\right)$ if $P \neq N P$. Later Engebretsen and Holmerin [5] improved this bound to $\Omega\left(2^{\sqrt{2 k}} / 2^{k}\right)$. For non-boolean MAX $k$-CSP $d$, Engebretsen [4] proved a hardness result of $2^{O(\sqrt{d})} / d^{k}$. Much stronger inapproximability results were obtained assuming the Unique Games Conjecture (UGC). Samorodnitsky and Trevisan [10] proved the hardness of $O\left(k / 2^{k}\right)$ for the boolean MAX $k$-CSP ${ }_{2}$. Austrin and Mossel [1] and, independently, Guruswami and Raghavendra [6] proved the hardness of $O\left(k d^{2} / d^{k}\right)$ for non-boolean MAX $k$-CSP ${ }_{d}$. Moreover, Austrin and Mossel [1] proved the hardness of $O\left(k d / d^{k}\right)$ for every $d$ and infinitely many $k$; specifically, their result holds for $d$ and $k$ such that $k=\left(d^{t}-1\right) /(d-1)$ for some $t \in \mathbb{N}$. Very recently, Håstad strengthened the result of Austrin and Mossel and showed the hardness of $O\left(k d / d^{k}\right)$ for every $d$ and $k \geq d$ [private communication].

On the positive side, approximation algorithms for the problem have been developed in a series of papers by Trevisan [12], Hast [7], Charikar, Makarychev and Makarychev [3], and Guruswami

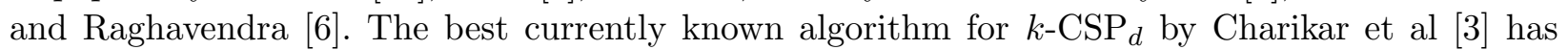
approximation factor of $\Omega\left(k \log d / d^{k}\right)$. Note that a trivial algorithm for MAX $k$-CSP ${ }_{d}$ that just picks a random assignment satisfies each constraint with probability at least $1 / d^{k}$, and therefore its approximation ratio is $1 / d^{k}$.

The problem is essentially settled in the boolean case. We know that the optimal approximation factor is $\Theta\left(k / 2^{k}\right)$ assuming UGC. However, best known lower and upper bounds for the non-boolean

\footnotetext{
* Yury Makarychev is supported in part by the NSF Career Award CCF-1150062.
} 
case do not match. In this paper, we present an approximation algorithm for non-boolean MAX $k$-CSP $d$ with approximation factor $\Omega\left(k d / d^{k}\right)$ (for $k \geq \Omega(\log d)$ ). This algorithm is asymptotically optimal assuming UGC - it is within a constant factor of the upper bounds of Austrin and Mossel and of Håstad (for $k$ of the form $\left(d^{t}-1\right) /(d-1)$ and for $k \geq d$, respectively). Our result improves the best previously known approximation factor of $\Omega\left(k \log d / d^{k}\right)$.

Related work Raghavendra studied a more general $\operatorname{MAX} \operatorname{CSP}(\mathcal{P})$ problem [8]. He showed that the optimal approximation factor equals the integrality gap of the standard SDP relaxation for the problem (assuming UGC). His result applies in particular to MAX $k$-CSP ${ }_{d}$. However, the SDP integrality gap of MAX $k-\mathrm{CSP}_{d}$ is not known.

Overview We use semidefinite programming (SDP) to solve the problem. In our SDP relaxation, we have an "indicator vector" $u_{i}$ for every variable $x_{u}$ and value $i$; we also have a "indicator vector" $z_{C}$ for every constraint $C$. In the intended solution, $u_{i}$ is equal to a fixed unit vector e if $x_{u}=i$, and $u_{i}=0$ if $x_{u} \neq i$; similarly, $z_{C}=\mathbf{e}$ if $C$ is satisfied, and $z_{C}=0$, otherwise.

It is interesting that the best previously known algorithm for the problem [3] did not use this SDP relaxation; rather it reduced the problem to a binary $k$-CSP problem, which it solved in turn using semidefinite programming. The only previously known algorithm [6] that directly rounded an SDP solution for MAX $k$-CSP ${ }_{d}$ had approximation factor $\Omega\left(\frac{k / d^{7}}{d^{k}}\right)$.

One of the challenges of rounding the SDP solution is that vectors $u_{i}$ might have different lengths. Consequently, we cannot just use a rounding scheme that projects vectors on a random direction and then chooses vectors that have largest projections, since this scheme will choose longer vectors with disproportionately large probabilities. To deal with this problem, we first develop a rounding scheme that rounds uniform SDP solutions, solutions in which all vectors are "short". Then we construct a randomized reduction that converts any instance to an instance with a uniform SDP solution.

Our algorithm for the uniform case is very simple. First, we choose a random Gaussian vector $g$. Then for every $u$, we find $u_{i}$ that has the largest projection on $g$ (in absolute value), and let $x_{u}=i$. However, the analysis of this algorithm is quite different from analyses of similar algorithms for other problems: when we estimate the probability that a constraint $C$ is satisfied, we have to analyze the correlation of all vectors $u_{i}$ with vector $z_{C}$ (where $\left\{u_{i}\right\}$ are SDP vectors for variables $x_{u}$ that appear in $C, z_{C}$ is the SDP vector for $C$ ), whereas the standard approach would be to look only at pairwise correlations of vectors $\left\{u_{i}\right\}$; this approach does not work in our case, however, since vectors corresponding to an assignment that satisfies $C$ may have very small pairwise correlations, but vectors corresponding to assignments that do not satisfy $C$ may have much larger pairwise correlations.

Remark 1.1. We study the problem only in the regime when $k \geq \Omega(\log d)$. In Theorem 5.1, we prove that when $k=O(\log d)$ our algorithm has approximation factor $e^{\Omega(k)} / d^{k}$. However, in this regime, a better approximation factor of $\Omega\left(d / d^{k}\right)$ can be obtained by a simple greedy approach.

Other Results We also apply our SDP rounding technique to the Boolean Maximum CSP Problem. We give an algorithm that has approximation guarantee $\approx 0.62 k / 2^{k}$ for sufficiently large $k$. That slightly improves the best previously known guarantee of $\approx 0.44 k / 2^{k}$ [3]. We present this result in Appendix B. 


\section{Preliminaries}

We apply the approximation preserving reduction of Trevisan [12] to transform a general instance of MAX $k-\mathrm{CSP}_{d}$ to an instance where each predicate is a conjunction of terms of the form $x_{u}=i$. The reduction replaces a predicate $P$, which depends on variables $x_{v_{1}}, \ldots, x_{v_{k}}$, with a set of clauses

$$
\left\{\left(x_{v_{1}}=i_{1}\right) \wedge \cdots \wedge\left(x_{v_{k}}=i_{k}\right): P\left(i_{1}, \ldots, i_{k}\right) \text { is true }\right\} .
$$

Then it is sufficient to solve the obtained instance. We refer the reader to [12] for details. We assume below that each predicate is a clause of the form $\left(x_{v_{1}}=i_{1}\right) \wedge \cdots \wedge\left(x_{v_{k}}=i_{k}\right)$.

Definition 2.1 (Constraint satisfaction problem). An instance $\mathcal{I}$ of $M A X C S P_{d}$ consists of

- a set of "indices" $X$,

- a set of variables $\left\{x_{u}\right\}_{u \in X}$ (there is one variable $x_{u}$ for every index $u \in X$ ),

- a set of clauses $\mathcal{C}$.

Each variable $x_{u}$ takes values in the domain $[d]=\{1, \ldots, d\}$. Each clause $C \in \mathcal{C}$ is a set of pairs $(u, i)$ where $u \in X$ and $i \in[d]$. An assignment $x_{u}=x_{u}^{*}$ satisfies a clause $C$ if for every $(u, i) \in C$, we have $x_{u}^{*}=i$. We assume that no clause $C$ in $\mathcal{C}$ contains pairs $(u, i)$ and $(u, j)$ with $i \neq j$ (no assignment satisfies such clause). The length of a clause $C$ is $|C|$. The support of $C$ is $\operatorname{supp}(C)=\{u:(u, i) \in C\}$.

The value of an assignment $x_{u}^{*}$ is the number of constraints in $\mathcal{C}$ satisfied by $x_{u}^{*}$. Our goal is to find an assignment of maximum value. We denote the value of an optimal assignment by $O P T=O P T(\mathcal{I})$.

In the $M A X k-C S P_{d}$ problem, we additionally require that all clauses in $\mathcal{C}$ have length at most $k$.

We consider the following semidefinite programming (SDP) relaxation for MAX $\mathrm{CSP}_{d}$. For every index $u \in X$ and $i \in[d]$, we have a vector variable $u_{i}$; for every clause $C$, we have a vector variable $z_{C}$.

$$
\begin{aligned}
& \text { maximize: } \sum_{C \in \mathcal{C}}\left\|z_{C}\right\|^{2} \\
& \text { subject to }
\end{aligned}
$$

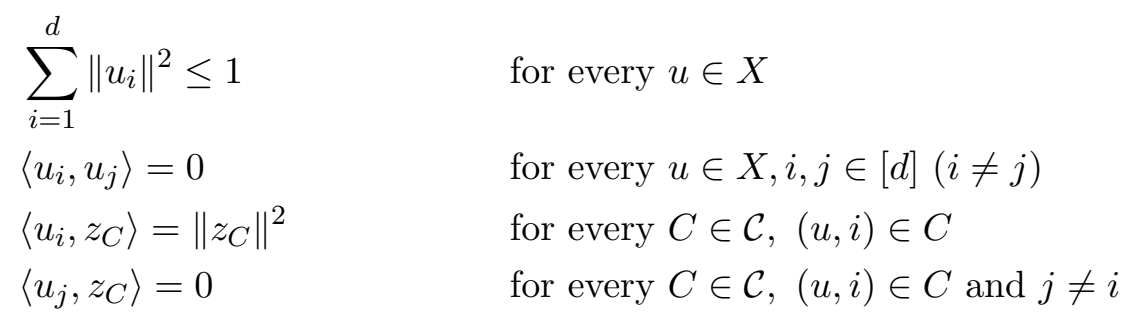

Denote the optimal SDP value by $S D P=S D P(\mathcal{I})$. Consider the optimal solution $x_{u}^{*}$ to an instance $\mathcal{I}$ and the corresponding SDP solution defined as follows,

$$
u_{i}=\left\{\begin{array}{ll}
\mathbf{e}, & \text { if } x_{u}^{*}=i ; \\
0, & \text { otherwise }
\end{array} \quad z_{C}= \begin{cases}\mathbf{e}, & \text { if } C \text { is satisfied } \\
0, & \text { otherwise }\end{cases}\right.
$$

where $\mathbf{e}$ is a fixed unit vector. It is easy to see that this is a feasible SDP solution and its value equals $O P T(\mathcal{I})$. Therefore, $\operatorname{SDP}(\mathcal{I}) \geq O P T(\mathcal{I})$. 
Definition 2.2. We say that an $S D P$ solution is uniform if $\left\|u_{i}\right\|^{2} \leq 1 / d$ for every $u \in X$ and $i \in[d]$.

Definition 2.3. Let $\xi$ be a standard Gaussian variable with mean 0 and variance 1 . We denote

$$
\begin{aligned}
& \Phi(t)=\operatorname{Pr}(|\xi| \leq t)=\frac{1}{\sqrt{2 \pi}} \int_{-t}^{t} e^{-x^{2} / 2} d x, \text { and } \\
& \bar{\Phi}(t)=1-\Phi(t)=\operatorname{Pr}(|\xi|>t) .
\end{aligned}
$$

We will use the following lemma, which we prove in Appendix.

Lemma 2.1. For every $t>0$ and $\beta \in(0,1]$, we have

$$
\bar{\Phi}(\beta t) \leq \bar{\Phi}(t)^{\beta^{2}} \text {. }
$$

We will also use the following result of Šidák [11]:

Theorem 2.1 (Šidák [11]). Let $\xi_{1}, \ldots, \xi_{r}$ be Gaussian random variables with mean zero and an arbitrary covariance matrix. Then for any positive $t_{1}, \ldots, t_{r}$,

$$
\operatorname{Pr}\left(\left|\xi_{1}\right| \leq t_{1},\left|\xi_{2}\right| \leq t_{2}, \ldots,\left|\xi_{r}\right| \leq t_{r}\right) \geq \prod_{i=1}^{r} \operatorname{Pr}\left(\left|\xi_{i}\right| \leq t_{i}\right) .
$$

\section{Rounding Uniform SDP Solutions}

In this section, we present a rounding scheme for uniform SDP solutions.

Lemma 3.1. There is a randomized polynomial-time algorithm that given an instance $\mathcal{I}$ of the $M A X C S P_{d}$ problem (with $d \geq 57$ ) and a uniform SDP solution, outputs an assignment $x_{u}$ such that for every clause $C \in \mathcal{C}$ :

$$
\operatorname{Pr}\left(C \text { is satisfied by } x_{u}\right) \geq \frac{\min \left(\left\|z_{C}\right\|^{2}|C| d / 8, e^{|C|}\right)}{2 d^{|C|}} .
$$

Proof. We use the following rounding algorithm:

\section{Rounding Scheme for Uniform SDP solutions}

Input: an instance of the MAX $\mathrm{CSP}_{d}$ problem and a uniform SDP solution.

Output: an assignment $\left\{x_{u}\right\}$.

- Choose a random Gaussian vector $g$ so that every component of $g$ is distributed as a Gaussian variable with mean 0 and variance 1 , and all components are independent.

- For every $u \in V$, let $x_{u}^{\prime}=\arg \max _{i}\left|\left\langle u_{i}, g\right\rangle\right|$.

- For every $u \in V$, choose $x_{u}^{\prime \prime}$ uniformly at random from $[d]$ (independently for different $u$ ).

- With probability $1 / 2$ return assignment $\left\{x_{u}^{\prime}\right\}$; with probability $1 / 2$ return assignment $\left\{x_{u}^{\prime \prime}\right\}$.

For every clause $C$, let us estimate the probabilities that assignments $x_{u}^{\prime}$ and $x_{u}^{\prime \prime}$ satisfy $C$. It is clear that $x_{u}^{\prime \prime}$ satisfies $C$ with probability $d^{-|C|}$. We prove now that $x_{u}^{\prime}$ satisfies $C$ with probability at least $d^{-3|C| / 4}$ if $\|z\|_{C}^{2} \geq 8 /(|C| d)$. 
Claim. Suppose $C \in \mathcal{C}$ is a clause such that $\|z\|_{C}^{2} \geq 8 /(|C| d)$ and $d \geq 57$. Then the probability that the assignment $x_{u}^{\prime}$ satisfies $C$ is at least $d^{-3|C| / 4}$.

Proof. Denote $s=|C|$. We assume without loss of generality that for every $u \in \operatorname{supp}(C),(u, 1) \in C$. Note that for $(u, i) \in C$, we have $\left\|z_{C}\right\|^{2}=\left\langle z_{C}, u_{i}\right\rangle \leq\left\|z_{C}\right\| \cdot\left\|u_{i}\right\| \leq\left\|z_{C}\right\| / \sqrt{d}$ (here we use that the SDP solution is uniform and therefore $\left.\left\|u_{i}\right\|^{2} \leq 1 / d\right)$. Thus $\left\|z_{C}\right\|^{2} \leq 1 / d$. In particular, $s=|C| \geq 8$ since $\|z\|_{C}^{2} \geq 8 /(|C| d)$.

For every $u \in \operatorname{supp}(C)$, let $u_{1}^{\perp}=u_{1}-z_{C}$. Let $\gamma_{u, 1}=\left\langle g, u_{1}^{\perp}\right\rangle$ and $\gamma_{u, i}=\left\langle g, u_{i}\right\rangle$ for $i \geq 2$. Let $\gamma_{C}=\left\langle g, z_{C}\right\rangle$. All variables $\gamma_{u, i}, \gamma_{C}$ are Gaussian variables. Using that for every two vectors $v$ and $w, \mathbb{E}[\langle g, v\rangle \cdot\langle g, w\rangle]=\langle v, w\rangle$, we get

$$
\begin{aligned}
& \mathbb{E}\left[\gamma_{C} \cdot \gamma_{u, 1}\right]=\left\langle z_{C}, u_{1}-z_{C}\right\rangle=\left\langle z_{C}, u_{1}\right\rangle-\left\|z_{C}\right\|^{2}=0 ; \\
& \mathbb{E}\left[\gamma_{C} \cdot \gamma_{u, i}\right]=\left\langle z_{C}, u_{i}\right\rangle=0 \text { for } i \geq 2 .
\end{aligned}
$$

Therefore, all variables $\gamma_{u, i}$ are independent from $\gamma_{C}$. (However, for $u^{\prime}, u^{\prime \prime} \in \operatorname{supp}(C)$ variables $\gamma_{u^{\prime}, i}$ and $\gamma_{u^{\prime \prime}, j}$ are not necessarily independent.) Let $M=\bar{\Phi}^{-1}\left(1 / d^{s / 2}\right) / \sqrt{s d / 8}$. We write the probability that $x_{u}^{\prime}$ satisfies $C$,

$$
\begin{aligned}
\operatorname{Pr}\left(x_{u}^{\prime} \text { satisfies } C\right)= & \operatorname{Pr}\left(\arg \max _{i}\left|\left\langle g, u_{i}\right\rangle\right|=1 \text { for every } u \in \operatorname{supp}(C)\right) \\
& =\operatorname{Pr}\left(\left|\left\langle g, u_{1}\right\rangle\right|>\left|\left\langle g, u_{i}\right\rangle\right| \text { for every } u \in \operatorname{supp}(C), i \in\{2, \ldots, d\}\right) \\
& =\operatorname{Pr}\left(\left|\gamma_{u, 1}+\gamma_{C}\right|>\left|\gamma_{u, i}\right| \text { for every } u \in \operatorname{supp}(C), i \in\{2, \ldots, d\}\right) \\
\geq & \operatorname{Pr}\left(\left|\gamma_{u, 1}\right| \leq M / 2, \text { and }\left|\gamma_{u, i}\right| \leq M / 2\right. \\
& \left.\quad \text { for every } u \in \operatorname{supp}(C), i \in\{2, \ldots, d\}|| \gamma_{C} \mid>M\right) \cdot \operatorname{Pr}\left(\left|\gamma_{C}\right|>M\right) .
\end{aligned}
$$

Since all variables $\gamma_{u, i}$ are independent from $\gamma_{C}$,

$\operatorname{Pr}\left(x_{u}^{\prime}\right.$ satisfies $\left.C\right) \geq \operatorname{Pr}\left(\left|\gamma_{u, i}\right| \leq M / 2\right.$ for every $\left.u \in \operatorname{supp}(C), i \in\{1, \ldots, d\}\right) \cdot \operatorname{Pr}\left(\left|\gamma_{C}\right|>M\right)$.

By Šidák's Theorem (Theorem 2.1), we have

$$
\operatorname{Pr}\left(x_{u}^{\prime} \text { satisfies } C\right) \geq\left(\prod_{u \in \operatorname{supp}(C)} \prod_{i=1}^{d} \operatorname{Pr}\left(\left|\gamma_{u, i}\right| \leq M / 2\right)\right) \cdot \operatorname{Pr}\left(\left|\gamma_{C}\right|>M\right) .
$$

We compute the variance of vectors $\gamma_{u, i}$. We use that $\operatorname{Var}[\langle g, v\rangle]=\|v\|^{2}$ for every vector $v$ and that the SDP solution is uniform.

$$
\begin{aligned}
& \operatorname{Var}\left[\gamma_{u, 1}\right]=\left\|u_{1}^{\perp}\right\|^{2}=\left\|u_{1}-z_{C}\right\|^{2}=\left\|u_{1}\right\|^{2}-2\left\langle u_{1}, z_{C}\right\rangle+\left\|z_{C}\right\|^{2}=\left\|u_{1}\right\|^{2}-\left\|z_{C}\right\|^{2} \leq\left\|u_{1}\right\|^{2} \leq 1 / d ; \\
& \operatorname{Var}\left[\gamma_{u, i}\right]=\left\|u_{i}\right\|^{2} \leq 1 / d \quad \text { for } i \geq 2 .
\end{aligned}
$$

Hence since $\Phi(t)$ is an increasing function and $\bar{\Phi}(\beta t) \leq \bar{\Phi}(t)^{\beta^{2}}$ (by Lemma 2.1), we have

$$
\begin{aligned}
\operatorname{Pr}\left(\left|\gamma_{u, i}\right| \leq M / 2\right) & =\Phi\left(M /\left(2 \sqrt{\operatorname{Var}\left[\gamma_{u, i}\right]}\right)\right) \geq \Phi(\sqrt{d} M / 2)=1-\bar{\Phi}(\sqrt{d} M / 2) \\
& \geq 1-\bar{\Phi}(\sqrt{s d / 8} M)^{2 / s}=1-\left(d^{-s / 2}\right)^{2 / s}=1-d^{-1}
\end{aligned}
$$

(recall that we defined $M$ so that $\bar{\Phi}(\sqrt{s d / 8} M)=d^{-s / 2}$ ). Similarly, $\operatorname{Var}\left[\gamma_{C}\right]=\left\|z_{C}\right\|^{2} \geq 8 /(s d)$ (by the condition of the lemma). We get (using the fact that $\bar{\Phi}(t)$ is a decreasing function),

$$
\operatorname{Pr}\left(\left|\gamma_{C}\right|>M\right)=\bar{\Phi}\left(M / \sqrt{\operatorname{Var}\left[\gamma_{C}\right]}\right) \geq \bar{\Phi}(M \sqrt{s d / 8})=d^{-s / 2} .
$$


Plugging in bounds for $\operatorname{Pr}\left(\left|\gamma_{u, i}\right| \leq M / 2\right)$ and $\operatorname{Pr}\left(\left|\gamma_{C}\right|>M\right)$ into (1), we obtain

$$
\operatorname{Pr}\left(x_{u}^{\prime} \text { satisfies } C\right) \geq\left(1-d^{-1}\right)^{d s} d^{-s / 2} \geq d^{-3 s / 4} .
$$

Here, we used that $\left(1-d^{-1}\right)^{d} \geq d^{-1 / 4}$ for $d \geq 57$ (the inequality $\left(1-d^{-1}\right)^{d} \geq d^{-1 / 4}$ holds for $d \geq 57$ since it holds for $d=57$ and the left hand side, $\left(1-d^{-1}\right)^{d}$, is an increasing function, the right hand side, $d^{-1 / 4}$, is a decreasing function).

We conclude that if $\left\|z_{C}\right\|^{2} \leq 8 /(|C| d)$ then the algorithm chooses assignment $x_{u}^{\prime \prime}$ with probability $1 / 2$ and this assignment satisfies $C$ with probability at least $1 / d^{|C|} \geq\left\|z_{C}\right\|^{2}|C| d /\left(8 d^{|C|}\right)$. So $C$ is satisfied with probability at least, $1 / d^{|C|} \geq\left\|z_{C}\right\|^{2}|C| d /\left(16 d^{|C|}\right)$; if $\left\|z_{C}\right\|^{2} \geq 8 /(|C| d)$ then the algorithm chooses assignment $x^{\prime}$ with probability $1 / 2$ and this assignment satisfies $C$ with probability at least $d^{-3|C| / 4} \geq e^{|C|} / d^{|C|}$ (since $\left.e \leq 57^{1 / 4} \leq d^{1 / 4}\right)$. In either case,

$$
\operatorname{Pr}(C \text { is satisfied }) \geq \frac{\min \left(\left\|z_{C}\right\|^{2}|C| d / 8, e^{|C|}\right)}{2 d^{|C|}} .
$$

Remark 3.1. We note that we did not try to optimize all constants in the statement of Lemma 3.1. By choosing all parameters in our proof appropriately, it is possible to show that for every constant $\varepsilon>0$, there is a randomized rounding scheme, $\delta>0$ and $d_{0}$ such that for every instance of MAX $\mathrm{CSP}_{d}$ with $d \geq d_{0}$ the probability that each clause $C$ is satisfied is at least $\min \left((1-\varepsilon)\left\|z_{C}\right\|^{2}\right.$. $\left.|C| d, \delta \cdot e^{\delta|C|}\right) / d^{|C|}$.

\section{Rounding Arbitrary SDP Solutions}

In this section, we show how to round an arbitrary SDP solution.

Lemma 4.1. There is a randomized polynomial-time algorithm that given an instance $\mathcal{I}$ of the $M A X C S P_{d}$ problem (with $d \geq 113$ ) and an SDP solution, outputs an assignment $x_{u}$ such that for every clause $C \in \mathcal{C}$ :

$$
\operatorname{Pr}\left(C \text { is satisfied by } x_{u}\right) \geq \frac{\min \left(\left\|z_{C}\right\|^{2}|C| d / 64,2 e^{|C| / 8}\right)}{4 d^{|C|}} .
$$

Proof. For every index $u$, we sort all vectors $u_{i}$ according to their length. Let $S_{u}$ be the indices of $\lceil d / 2\rceil$ shortest vectors among $u_{i}$, and $L_{u}=[d] \backslash S_{u}$ be the indices of $\lfloor d / 2\rfloor$ longest vectors among $u_{i}$ (we break ties arbitrarily). For every clause $C$ let $r(C)=\left|\left\{(u, i) \in C: i \in S_{u}\right\}\right|$.

Claim. For every $i \in S_{u}$, we have $\left\|u_{i}\right\|^{2} \leq 1 /\left|S_{u}\right|$.

Proof. Let $i \in S_{u}$. Note that $\left\|u_{i}\right\|^{2}+\sum_{j \in L_{u}}\left\|u_{j}\right\|^{2} \leq 1$ (this follows from SDP constraints). There are at least $\lceil d / 2\rceil$ terms in the sum, and $\left\|u_{i}\right\|^{2}$ is the smallest among them (since $i \in S_{u}$ ). Thus $\left\|u_{i}\right\|^{2} \leq 1 /\lceil d / 2\rceil=1 /\left|S_{u}\right|$.

We use a combination of two rounding schemes: one of them works well on clauses $C$ with $r(C) \geq|C| / 4$, the other on clauses $C$ with $r(C) \leq|C| / 4$. 
Lemma 4.2. There is a polynomial-time randomized rounding algorithm that given an $M A X C S P_{d}$ instance $\mathcal{I}$ with $d \geq 113$ outputs an assignment $x_{u}$ such that every clause $C$ with $r(C) \geq|C| / 4$ is satisfied with probability at least

$$
\frac{\min \left(\left\|z_{C}\right\|^{2}|C| d / 64, e^{|C| / 4}\right)}{2 d^{|C|}}
$$

Proof. We will construct a sub-instance $\mathcal{I}^{\prime}$ with a uniform SDP solution and then solve $\mathcal{I}^{\prime}$ using Lemma 3.1. To this end, we first construct a partial assignment $x_{u}$. For every $u \in X$, with probability $\left|L_{u}\right| / d=\lfloor d / 2\rfloor / d$, we assign a value to $x_{u}$ uniformly at random from $L_{u}$; with probability $1-$ $\left|L_{u}\right| / d=\left|S_{u}\right| / d$, we do not assign any value to $x_{u}$. Let $A=\left\{u: x_{u}\right.$ is assigned $\}$. Let us say that a clause $C$ survives the partial assignment step if for every $(u, i) \in C$ either $u \in A$ and $i=x_{u}$, or $u \notin A$ and $i \in S_{u}$.

The probability that a clause $C$ survives is

$$
\begin{gathered}
\prod_{(u, i) \in C, i \in L_{u}} \operatorname{Pr}\left(x_{u} \text { is assigned value } i\right) \prod_{(u, i) \in C, i \in S_{u}} \operatorname{Pr}\left(x_{u} \text { is unassigned }\right)= \\
\left(\frac{\lfloor d / 2\rfloor}{d} \cdot \frac{1}{\lfloor d / 2\rfloor}\right)^{|C|-r(C)} \cdot\left(\frac{\lceil d / 2\rceil}{d}\right)^{r(C)}=\frac{\lceil d / 2\rceil^{r(C)}}{d^{|C|}} .
\end{gathered}
$$

For every survived clause $C$, let $C^{\prime}=\{(u, i): u \notin A\}$. Note that for every $(u, i) \in C^{\prime}$, we have $i \in S_{u}$. We get a sub-instance $\mathcal{I}^{\prime}$ of our problem on the set of unassigned variables $\left\{x_{u}: u \notin A\right\}$ with the set of clauses $\left\{C^{\prime}: C \in \mathcal{C}\right.$ survives $\}$. The length of each clause $C^{\prime}$ equals $r(C)$. In sub-instance $\mathcal{I}^{\prime}$, we require that each variable $x_{u}$ takes values in $S_{u}$. Thus $\mathcal{I}^{\prime}$ is an instance of MAX $\operatorname{CSP}_{d^{\prime}}$ problem with $d^{\prime}=\left|S_{u}\right|=\lceil d / 2\rceil$.

Now we transform the SDP solution for $\mathcal{I}$ to an SDP solution for $\mathcal{I}^{\prime}$ : we let $z_{C^{\prime}}=z_{C}$ for survived clauses $C$, remove vectors $u_{i}$ for all $u \in A, i \in[d]$ and remove vectors $z_{C}$ for non-survived clauses $C$. By Claim 4, this SDP solution is a uniform solution for $\mathcal{I}^{\prime}$ (i.e. $\left\|u_{i}\right\| \leq 1 / d^{\prime}$ for every $u \notin A$ and $i \in S_{i}$; note that $\mathcal{I}^{\prime}$ has alphabet size $\left.d^{\prime}\right)$. We run the rounding algorithm from Lemma 3.1. The algorithm assigns values to unassigned variables $x_{u}$. For every survived clause $C$, we get

$$
\begin{aligned}
\operatorname{Pr}\left(C \text { is satisfied by } x_{u}\right) & =\operatorname{Pr}\left(C^{\prime} \text { is satisfied by } x_{u}\right) \geq \frac{\min \left(\left\|z_{C}\right\|^{2}\left|C^{\prime}\right| d^{\prime} / 8, e^{\left|C^{\prime}\right|}\right)}{2 d^{\prime\left|C^{\prime}\right|}} \\
& =\frac{\min \left(\left\|z_{C}\right\|^{2} r(C) d^{\prime} / 8, e^{r(C)}\right)}{2 d^{\prime r(C)}} \geq \frac{\min \left(\left\|z_{C}\right\|^{2}|C| d / 64, e^{|C| / 4}\right)}{2 d^{\prime r(C)}} .
\end{aligned}
$$

Therefore, for every clause $C$,

$\operatorname{Pr}\left(C\right.$ is satisfied by $\left.x_{u}\right) \geq \operatorname{Pr}\left(C\right.$ is satisfied by $x_{u} \mid C$ survives $) \operatorname{Pr}(C$ survives $)$

$$
\begin{aligned}
& \geq \frac{\min \left(\left\|z_{C}\right\|^{2}|C| d / 64, e^{|C| / 4}\right)}{2 d^{\prime r(C)}} \times \frac{\lceil d / 2\rceil^{r(C)}}{d^{|C|}} \\
& =\frac{\min \left(\left\|z_{C}\right\|^{2}|C| d / 64, e^{|C| / 4}\right)}{2 d^{|C|}} .
\end{aligned}
$$

Finally, we describe an algorithm for clauses $C$ with $r(C) \leq|C| / 4$. 
Lemma 4.3. There is a polynomial-time randomized rounding algorithm that given an $M A X C S P_{d}$ instance $\mathcal{I}$ outputs an assignment $x_{u}$ such that every clause $C$ with $r(C) \leq|C| / 4$ is satisfied with probability at least $e^{|C| / 8} / d^{|C|}$.

Proof. We do the following independently for every vertex $u \in X$. With probability $3 / 4$, we choose $x_{u}$ uniformly at random from $L_{u}$; with probability $1 / 4$, we choose $x_{u}$ uniformly at random from $S_{u}$. The probability that a clause $C$ with $r(C) \leq|C| / 4$ is satisfied equals

$$
\begin{aligned}
\prod_{(u, i) \in C, i \in L_{u}} \frac{3}{4\left|L_{u}\right|} & \prod_{(u, i) \in C, i \in S_{u}} \frac{1}{4\left|S_{u}\right|}=\frac{1}{d^{|C|}} \cdot\left(\frac{3 d}{4\left|L_{u}\right|}\right)^{|C|-r(C)}\left(\frac{d}{4\left|S_{u}\right|}\right)^{r(C)} \\
& \geq \frac{1}{d^{|C|}} \cdot\left(\frac{3 d}{4\left|L_{u}\right|}\right)^{3|C| / 4}\left(\frac{d}{4\left|S_{u}\right|}\right)^{|C| / 4} \geq \frac{1}{d^{|C|}} \cdot\left(\left(\frac{3}{2}\right)^{3 / 4}\left(\frac{d}{2(d+1)}\right)^{1 / 4}\right)^{|C|} .
\end{aligned}
$$

Note that $\left(\frac{3}{2}\right)^{3 / 4}\left(\frac{d}{2(d+1)}\right)^{1 / 4} \geq\left(\frac{3}{2}\right)^{3 / 4}\left(\frac{113}{2 \cdot 114}\right)^{1 / 4} \geq e^{1 / 8}$. Therefore, the probability that the clause is satisfied is at least $e^{|C| / 8} / d^{|C|}$.

We run the algorithm from Lemma 4.2 with probability $1 / 2$ and the algorithm from Lemma 4.3 with probability $1 / 2$. Consider a clause $C \in \mathcal{C}$. If $r(C) \geq|C| / 4$, we satisfy $C$ with probability at

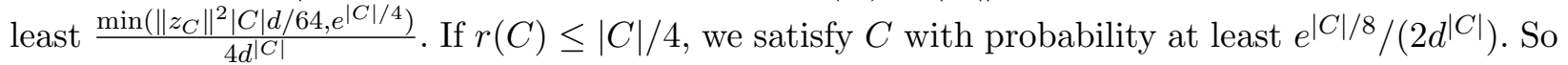
we satisfy every clause $C$ with probability at least $\frac{\min \left(\left\|z_{C}\right\|^{2}|C| d / 64,2 e^{|C| / 8}\right)}{4 d^{|C|}}$.

\section{Approximation Algorithm for MAX $k-\mathrm{CSP}_{d}$}

In this section, we present the main result of the paper.

Theorem 5.1. There is a polynomial-time randomized approximation algorithm for $M A X k$-CSP $P_{d}$ that given an instance $\mathcal{I}$ finds an assignment that satisfies at least $\Omega\left(\min \left(k d, e^{k / 8}\right) O P T(\mathcal{I}) / d^{k}\right)$ clauses with constant probability.

Proof. If $d \leq 113$, we run the algorithm of Charikar, Makarychev and Makarychev [3] and get $\Omega\left(k / d^{k}\right)$ approximation. So we assume below that $d \geq 113$. We also assume that $k d / d^{k} \geq 1 /|\mathcal{C}|$, as otherwise we just choose one clause from $\mathcal{C}$ and find an assignment that satisfies it. Thus $d^{k}$ is polynomial in the size of the input.

We solve the SDP relaxation for the problem and run the rounding scheme from Lemma $4.1 d^{k}$ times. We output the best of the obtained solutions. By Lemma 4.1, each time we run the rounding scheme we get a solution with expected value at least

$$
\begin{aligned}
\sum_{C \in \mathcal{C}} \frac{\min \left(\left\|z_{C}\right\|^{2}|C| d / 64,2 e^{|C| / 8}\right)}{4 d^{|C|}} & \geq \sum_{C \in \mathcal{C}} \frac{\min \left(k d / 64,2 e^{k / 8}\right)}{4 d^{k}}\left\|z_{C}\right\|^{2} \geq \frac{\min \left(k d / 64,2 e^{k / 8}\right)}{4 d^{k}} S D P(\mathcal{I}) \\
& \geq \frac{\min \left(k d / 64,2 e^{k / 8}\right)}{4 d^{k}} O P T(\mathcal{I}) .
\end{aligned}
$$

Denote $\alpha=\frac{\min \left(k d / 64,2 e^{k / 8}\right)}{4 d^{k}}$. Let $Z$ be the random variable equal to the number of satisfied clauses. Then $\mathbb{E}[Z] \geq \alpha O P T(\mathcal{I})$, and $Z \leq O P T(\mathcal{I})$ (always). Let $p=\operatorname{Pr}(Z \leq \alpha O P T(\mathcal{I}) / 2)$. Then

$$
p \cdot(\alpha O P T(\mathcal{I}) / 2)+(1-p) \cdot O P T(\mathcal{I}) \geq \mathbb{E}[Z] \geq \alpha O P T(\mathcal{I}) .
$$


So $p \leq \frac{1-\alpha}{1-\alpha / 2}=1-\frac{\alpha}{2-\alpha}$. So with probability at least $1-p \geq \frac{\alpha}{2-\alpha}$, we find a solution of value at least $\alpha O P T(\mathcal{I}) / 2$ in one iteration. Since we perform $d^{k}>1 / \alpha$ iterations, we find a solution of value at least $\alpha O P T(\mathcal{I}) / 2$ with constant probability.

\section{References}

1. P. Austrin and E. Mossel. Approximation Resistant Predicates from Pairwise Independence. Computational Complexity, 18(2):249271, 2009.

2. M. Charikar, K. Makarychev, and Y. Makarychev. Near-Optimal Algorithms for Unique Games. In Proceedings of the 38th ACM Symposium on Theory of Computing, pp. 205-214, 2006.

3. M. Charikar, K. Makarychev, and Y. Makarychev. Near-Optimal Algorithms for Maximum Constraint Satisfaction Problems. ACM Transactions on Algorithms, 5(3), July 2009.

4. L. Engebretsen. The Nonapproximability of Non-Boolean Predicates. SIAM Journal on Discrete Mathematics, 18(1), pp. 114129, 2004.

5. L. Engebretsen and J. Holmerin. More Efficient Queries in PCPs for NP and Improved Approximation Hardness of Maximum CSP. In Proceedings of the Symposium on Theoretical Aspects of Computer Science (STACS), pp. 194-205, 2005.

6. V. Guruswami and P. Raghavendra. Constraint Satisfaction over a Non-Boolean Domain: Approximation Algorithms and Unique-Games Hardness. In Proceedings of APPROX 2008, pp. 77-90, 2008.

7. G. Hast. Approximating Max $k \mathrm{CSP}$ - Outperforming a Random Assignment with Almost a Linear Factor. In Proceedings of the 32nd International Colloquium on Automata, Languages and Programming, pp. 956-968, 2005.

8. P. Raghavendra. Optimal Algorithms and Inapproximability Results For Every CSP? In Proceeding of the ACM Symposium on Theory of Computing (STOC), 2008.

9. A. Samorodnitsky and L. Trevisan. A PCP characterization of NP with optimal amortized query complexity. In Proceedings of the ACM Symposium on Theory of Computing (STOC), pp. 191-199, 2000.

10. A. Samorodnitsky and L. Trevisan. Gowers Uniformity, Influence of Variables, and PCPs. In Proceedings of the 38th ACM symposium on Theory of computing, pp. 11-20, 2006.

11. Z. Šidák. Rectangular Confidence Regions for the Means of Multivariate Normal Distributions. Journal of the American Statistical Association, vol. 62, no. 318, pp. 626-633, Jun. 1967.

12. L. Trevisan. Parallel Approximation Algorithms by Positive Linear Programming. Algorithmica, vol. 21, no. 1, pp. 72-88, 1998.

\section{A Proof of Lemma 2.1}

In this section, we prove Lemma 2.1. We will use the following fact.

Lemma A.1. [see e.g. [2]] For every $t>0$,

$$
\frac{2 t}{\sqrt{2 \pi}\left(t^{2}+1\right)} e^{-\frac{t^{2}}{2}}<\bar{\Phi}(t)<\frac{2}{\sqrt{2 \pi} t} e^{-\frac{t^{2}}{2}} .
$$

Lemma 2.1. For every $t>0$ and $\beta \in(0,1]$, we have

$$
\bar{\Phi}(\beta t) \leq \bar{\Phi}(t)^{\beta^{2}} .
$$

Proof. Rewrite the inequality we need to prove as follows: $(\bar{\Phi}(\beta t))^{1 / \beta^{2}} \leq \bar{\Phi}(t)$. Denote the left hand side by $f(\beta, t)$ :

$$
f(\beta, t)=\bar{\Phi}(\beta t)^{1 / \beta^{2}}
$$

We show that for every $t>0, f(\beta, t)$ is strictly increasing function as a function of $\beta \in(0,1]$. Then,

$$
(\bar{\Phi}(\beta t))^{1 / \beta^{2}}=f(\beta)<f(1)=\bar{\Phi}(t) .
$$


We first prove that $\frac{\partial f(1, t)}{\partial \beta}>0$. Write,

$$
\frac{\partial f(1, t)}{\partial \beta}=-2 \log (\bar{\Phi}(t)) \bar{\Phi}(t)+t \bar{\Phi}^{\prime}(t)=-2 \log (\bar{\Phi}(t)) \bar{\Phi}(t)-\frac{2 t e^{-t^{2} / 2}}{\sqrt{2 \pi}} .
$$

Consider three cases. If $t \geq \sqrt{\frac{2 e}{\pi}}$, then, by Lemma A.1,

$$
\bar{\Phi}(t)<\frac{2}{\sqrt{2 \pi} t} e^{-t^{2} / 2} \leq e^{-1 / 2} e^{-t^{2} / 2}=e^{-\left(t^{2}+1\right) / 2} .
$$

Hence, $-2 \log (\bar{\Phi}(t))>\left(t^{2}+1\right)$, and by Lemma A.1,

$$
-2 \log (\bar{\Phi}(t)) \bar{\Phi}(t)>\left(t^{2}+1\right) \bar{\Phi}(t)>\frac{2 t e^{-t^{2} / 2}}{\sqrt{2 \pi}} .
$$

If $t<\sqrt{\frac{2 e}{\pi}}$, then let $\rho(x)=-\log x /(1-x)$ for $x \in(0,1)$ and write,

$$
-\log \bar{\Phi}(t)=\rho(\bar{\Phi}(t)) \cdot(1-\bar{\Phi}(t))=\frac{\rho(\bar{\Phi}(t))}{\sqrt{2 \pi}} \int_{-t}^{t} e^{-x^{2} / 2} d x \geq \frac{2 \rho(\bar{\Phi}(t)) t e^{-t^{2} / 2}}{\sqrt{2 \pi}}
$$

Hence,

$$
\frac{\partial f(1, t)}{\partial \beta}=-2 \log (\bar{\Phi}(t)) \bar{\Phi}(t)-\frac{2 t e^{-t^{2} / 2}}{\sqrt{2 \pi}} \geq \frac{2 t e^{-t^{2} / 2}}{\sqrt{2 \pi}} \times(2 \rho(\bar{\Phi}(t)) \bar{\Phi}(t)-1) .
$$

For $x \in[1 / 3,1], 2 \rho(x) x>1$, since the function $\rho(x) x$ is increasing and $\rho(1 / 3)>3 / 2$. Hence $2 \rho(\bar{\Phi}(t)) \bar{\Phi}(t)>1$, if $\bar{\Phi}(t) \geq 1 / 3$.

The remaining case is $t<\sqrt{\frac{2 e}{\pi}}$ and $\bar{\Phi}(t)<1 / 3$. Then, $\bar{\Phi}(t) \geq \bar{\Phi}\left(\sqrt{\frac{2 e}{\pi}}\right)>1 / 6$ and hence $\bar{\Phi}(t) \in(1 / 6,1 / 3)$. Since the function $-x \log x$ is increasing on the interval $\left(0, e^{-1}\right)$,

$$
-2 \log (\bar{\Phi}(t)) \bar{\Phi}(t)>-2 \log (1 / 6) \cdot \frac{1}{6}>\frac{1}{2} .
$$

The function $t e^{-t^{2} / 2}$ attains its maximum at $t=1$, thus

$$
\frac{2 t e^{-t^{2} / 2}}{\sqrt{2 \pi}} \leq \frac{2 e^{-1 / 2}}{\sqrt{2 \pi}}<\frac{1}{2}
$$

We get

$$
\frac{\partial f(1, t)}{\partial \beta}=-2 \log (\bar{\Phi}(t)) \bar{\Phi}(t)-\frac{2 t e^{-t^{2} / 2}}{\sqrt{2 \pi}}>0 .
$$

Since $\frac{\partial f(1, t)}{\partial \beta}>0$, for every $t>0$, there exists $\varepsilon_{0}>0$, such that for all $\varepsilon \in\left(0, \varepsilon_{0}\right), f(1-\varepsilon, t)<$ $f(1, t)$. Particularly, for $t^{\prime}=\beta t$,

$$
f(\beta, t)=f\left(1, t^{\prime}\right)^{1 / \beta^{2}} \geq f\left(1-\varepsilon, t^{\prime}\right)^{1 / \beta^{2}}=f((1-\varepsilon) \beta, t) .
$$




\section{B Improved Approximation Factor for Boolean Max $k$-CSP}

In this section, we present an approximation algorithm for the boolean Maximum $k$-CSP problem, MAX $k-\mathrm{CSP}_{2}$. The algorithm has approximation factor $0.626612 k / 2^{k}$ if $k$ is sufficiently large. This bound improves the previously best known bound of $0.44 k / 2^{k}$ [3] (if $k$ is sufficiently large).

Our algorithm is a slight modification of the algorithm for rounding uniform solutions of MAX $k$ - $\mathrm{CSP}_{d}$. We use the SDP relaxation presented in Section 2. Without loss of generality, we will assume below that all clauses have length exactly $k$. If a clause $C$ is shorter, we can introduce $k-|C|$ new variables and append them to $C$. This transformation will not change the value of the instance.

First, we describe a rounding scheme for an SDP solution $\left\{u_{1}, u_{2}\right\}_{u \in X} \cup\left\{z_{C}\right\}_{C \in \mathcal{C}}$.

Lemma B.1. There is a polynomial-time randomized rounding algorithm such that for every clause $C \in \mathcal{C}$ the probability that the algorithm satisfies $C$ is at least

$$
\frac{1}{2^{k} \sqrt{2 \pi k}} \int_{0}^{\infty} h_{\beta}(t)^{k} d t, \text { where } h_{\beta}(t)=2 \Phi(\beta t) e^{-t^{2} / 2},
$$

and $\beta=\sqrt{k}\left\|z_{C}\right\|_{2}$.

Proof. We round the SDP solution as follows.

\section{SDP Rounding Scheme for MAX $k-\mathrm{CSP}_{2}$}

Input: an instance of MAX $k-\mathrm{CSP}_{2}$ and an SDP solution.

Output: an assignment $\left\{x_{u}\right\}$.

- Choose a random Gaussian vector $g$ so that every component of $g$ is distributed as a Gaussian variable with mean 0 and variance 1 , and all components are independent.

- For every $u \in V$, let $x_{u}=\arg \max _{i}\left\langle u_{i}, g\right\rangle$.

Consider a clause $C \in \mathcal{C}$. We assume without loss of generality that $C=\{(u, 1): u \in \operatorname{supp}(C)\}$. Let $\gamma_{C}=\left\langle z_{C}, g\right\rangle$ and $\gamma_{u}=\left\langle u_{2}-u_{1}+z_{C}, g\right\rangle$ for $u \in \operatorname{supp}(C)$. Note that for $u \in \operatorname{supp}(C)$,

$$
\begin{aligned}
\operatorname{Var}\left[\gamma_{C}\right] & =\left\|z_{C}\right\|^{2}=\beta^{2} / k, \\
\operatorname{Var}\left[\gamma_{u}\right] & =\left\|u_{2}-u_{1}+z_{C}\right\|^{2}=\left\|u_{1}\right\|+\left\|u_{2}\right\|^{2}+\left\|z_{C}\right\|^{2}-2\left\langle u_{1}, z_{C}\right\rangle=\left\|u_{1}\right\|+\left\|u_{2}\right\|^{2}-\left\|z_{C}\right\|^{2} \leq 1, \\
\mathbb{E}\left[\gamma_{C} \gamma_{u}\right] & =\left\langle z_{C}, u_{2}-u_{1}+z_{C}\right\rangle=\left\langle z_{C}, u_{2}\right\rangle-\left\langle z_{C}, u_{1}\right\rangle+\left\langle z_{C}, z_{C}\right\rangle=0-\left\|z_{C}\right\|^{2}+\left\|z_{C}\right\|^{2}=0 .
\end{aligned}
$$

Therefore, all random variables $\gamma_{u}$, for $u \in \operatorname{supp} C$, are independent from $\gamma_{C}$. The probability that $C$ is satisfied equals

$$
\begin{aligned}
\operatorname{Pr}(C \text { is satisfied }) & =\operatorname{Pr}\left(\left\langle u_{1}, g\right\rangle>\left\langle u_{2}, g\right\rangle \text { for every } u \in \operatorname{supp}(C)\right) \\
& =\operatorname{Pr}\left(\gamma_{C}>\gamma_{u} \text { for every } u \in \operatorname{supp}(C)\right) \geq \operatorname{Pr}\left(\left|\gamma_{u}\right|<\gamma_{C} \text { for every } u \in \operatorname{supp}(C)\right) \\
& =\mathbb{E}_{\gamma_{C}}\left[\operatorname{Pr}\left(\left|\gamma_{u}\right| \leq \gamma_{C} \text { for every } u \in \operatorname{supp}(C) \mid \gamma_{C}\right)\right] \\
& \stackrel{\text { let } t=\gamma_{C} / \beta}{=} \frac{1}{\sqrt{2 \pi k}} \int_{t=0}^{\infty} \operatorname{Pr}\left(\left|\gamma_{u}\right| \leq \beta t \text { for every } u \in \operatorname{supp}(C)\right) e^{-k t^{2} / 2} d t .
\end{aligned}
$$


We use here that $\operatorname{Var}\left[\gamma_{C} / \beta\right]=1 / k$. By Šidák's Theorem (Theorem 2.1), we have

$$
\begin{aligned}
\operatorname{Pr}\left(\left|\gamma_{u}\right| \leq \beta t \text { for every } u \in \operatorname{supp}(C)\right) & \geq \prod_{u \in \operatorname{supp}(C)} \operatorname{Pr}\left(\left|\gamma_{u}\right| \leq \beta t\right)=\prod_{u \in \operatorname{supp}(C)} \Phi\left(\beta t / \sqrt{\operatorname{Var}\left[\gamma_{u}\right]}\right) \\
& \geq \prod_{u \in \operatorname{supp}(C)} \Phi(\beta t)=\Phi(\beta t)^{k} .
\end{aligned}
$$

We conclude that

$$
\operatorname{Pr}(C \text { is satisfied }) \geq \frac{1}{2^{k} \sqrt{2 \pi k}} \int_{0}^{\infty} h_{\beta}(t)^{k} d t
$$

Let $g(\beta)=\max _{t \in \mathbb{R}} h_{\beta}(t)\left(h_{\beta}(\mathrm{t})\right.$ attains its maximum since $h_{\beta}(t) \rightarrow 0$ as $\left.t \rightarrow \infty\right)$. Note that $g(\beta)$ is an increasing function since $h_{\beta}(t)$ is an increasing function of $\beta$ for every fixed $t$. Additionally, $g(0)=0$ and $\lim _{\beta \rightarrow \infty} g(\beta)=2$ since $f(\beta, 1 / \sqrt{\beta})=2 \Phi(\sqrt{\beta}) e^{-1 /(2 \beta)} \rightarrow 2$ as $\beta \rightarrow \infty$, and for every $\beta$ and $t, f(\beta, t) \leq 2$. Therefore, $\beta^{-1}$ is defined on $[0,2)$. Let $\beta_{0}=g^{-1}(1)$. It is easy to check numerically that $\beta_{0} \in(1.263282,1.263283)$.

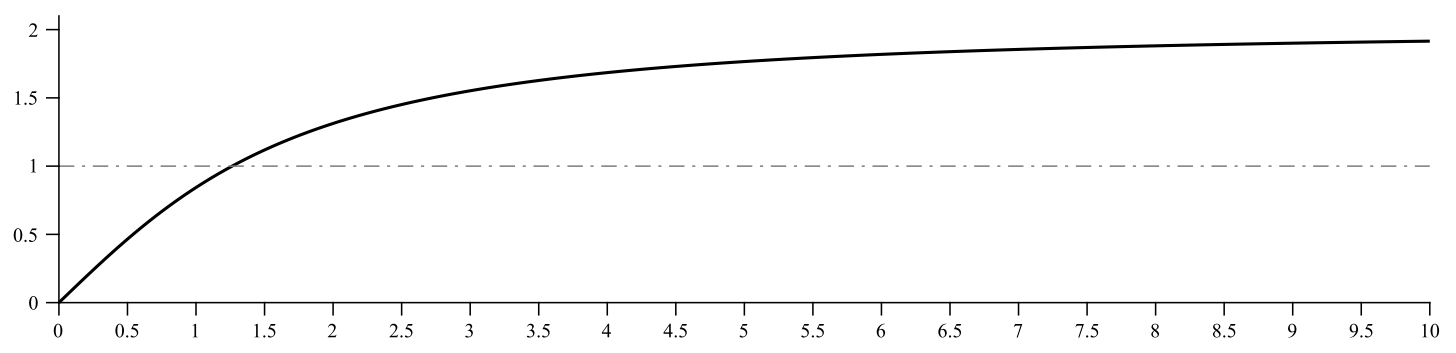

Fig. 1. The figure shows the graph of $g(t)$. We note that $g(t)>1$ when $t>\beta_{0} \approx 1.263282$.

Claim. For every $\beta>\beta_{0}$ there exists $k_{0}$ (which depends only on $\beta$ ) such that if $k \geq k_{0}$ and $\left\|z_{C}\right\| \geq \beta / \sqrt{k}$ then the probability that the algorithm from Lemma B.1 returns an assignment that satisfies $C$ is at least $k^{2} / 2^{k}$.

Proof. Let $\varepsilon_{1}=(g(\beta)-1) / 2>0$. Let $\varepsilon_{2}$ be the measure of the set $\left\{t: h_{\beta}(t)>1+\varepsilon_{1}\right\}$. Since $h_{\beta}(t)$ is continuous, $\varepsilon_{2}>0$.

The probability that $C$ is satisfied is at least

$$
\frac{1}{2^{k} \sqrt{2 \pi k}} \int_{0}^{\infty} h_{\beta}(t)^{k} d t \geq \frac{\varepsilon_{2}\left(1+\varepsilon_{1}\right)^{k}}{2^{k} \sqrt{2 \pi k}} .
$$

We choose $k_{0}$ so that for every $k \geq k_{0}$

$$
\varepsilon_{2}\left(1+\varepsilon_{1}\right)^{k} \geq \sqrt{2 \pi k} \cdot k^{2} .
$$

Then if $k \geq k_{0}$ the probability that the clause is satisfied is at least $k^{2} / 2^{k}$.

Now we are ready to describe our algorithm. 
Theorem B.1. There is a randomized approximation algorithm for the boolean MAX k-CSP problem with approximation guarantee $\alpha_{k} k / 2^{k}$ where $\alpha_{k} \rightarrow \alpha_{0} \geq 0.626612$ as $k \rightarrow \infty$ and $\alpha_{0}=1 / \beta_{0}^{2}$. (Here, as above, $\beta_{0}$ is the solution of the equation $g(\beta)=1$ where $g(\beta)=\max _{t \in \mathbb{R}} 2 \Phi(\beta t) e^{-t^{2} / 2}$.)

Proof. The algorithm with probability $p=1 / k$ rounds the SDP solution as described in Lemma B.1, with probability $1-p$, it choses a completely random solution.

Let $\alpha<\alpha_{0}$. We will show that if $k$ is large enough, every clause is satisfied with probability at least $\alpha k / 2^{k}$. Let $\beta=\left(\beta_{0}+\alpha^{-1 / 2}\right) / 2 \in\left(\beta_{0}, \alpha^{-1 / 2}\right)$. Let $k_{0}$ be as in Claim B. Suppose that $k \geq \max \left(k_{0},\left(1-\alpha \beta^{2}\right)^{-1}\right)$.

Consider a clause $C$. We show that the algorithm satisfies $C$ with probability at least $\frac{\alpha\left\|z_{C}\right\|^{2} k}{2^{k}}$. Indeed, we have:

- If $\left\|z_{C}\right\|<\beta / \sqrt{k}$, the clause is satisfied with probability at least $(1-p) / 2^{k} \geq \frac{(1-p) k\left\|z_{C}\right\|^{2}}{\beta^{2} 2^{k}} \geq$ $\alpha\left\|z_{C}\right\|^{2} k / 2^{k}$.

- If $\left\|z_{C}\right\| \geq \beta / \sqrt{k}$, the clause is satisfied with probability at least $p \cdot k^{2} / 2^{k}=k / 2^{k} \geq k\left\|z_{C}\right\|^{2} / 2^{k}$.

We conclude that the algorithm finds a solution that satisfies at least

$$
\frac{\alpha k}{2^{k}} \sum_{C \in \mathcal{C}}\left\|z_{C}\right\|^{2}=\frac{\alpha k}{2^{k}} \cdot S D P \geq \frac{\alpha k}{2^{k}} \cdot O P T
$$

clauses in expectation. By running this algorithm polynomially many times (as we do in Theorem 5.1) we can find a solution of value at least $\alpha^{\prime} k O P T / 2^{k}$ for every constant $\alpha^{\prime}<\alpha$ w.h.p. 